\title{
Some Properties of the Prolongation Limit Random Sets in Random Dynamical Systems
}

\author{
Sundus Talib Mohsin Ihsan Jabbar Kadhim \\ College of Computer Science and Mathematics, University of Al-Qadiysih \\ sundustalibm@gmail.com \\ Ihsan.kadhim@qu.edu.iq
}

Recived : $18 \backslash 10 \backslash 2018$

Revised : $11 \backslash 11 \backslash 2018$

Accepted : $12 \backslash 12 \backslash 2018$

Available online : $\quad 28 / 1 / 2019$

DOI: $10.29304 / j q c m .2019 .11 .1 .476$

\begin{abstract}
:
The aim of this paper is to study the omega limit set with new concepts of the prolongation limit random sets in random dynamical systems, where some properties are proved and introduced such as the relation among the orbit closure, orbit and omega limit random set. Also we prove that the first prolongation of a closed random set containing this set, the first prolongation is closed and invariant. In addition, it is connected whenever it is compact provided that the phase space of the random dynamical systems is locally compact. Then, we study the prolongational limit random set and examined some essential properties of this set. Finally, the relation among the first prolongation, the prolongational limit random set and the positive trajectory of a random set is given and proved.
\end{abstract}

Keywords: random dynamical system, trajectories,Omega-Limi set, prolongations and prolongational limit of random dynamical system.

Mathematics Subject Classification: 37HXX. 


\section{Introduction.}

Random dynamical systems arise in the modeling of many phenomena in physics, biology, economics, climatology, etc. , and the random effects often reflect intrinsic properties of these phenomena rather than just to compensate for the defects in deterministic models. The history of study of random dynamical systems goes back to Ulam and von Neumann in 1945 [1] and it has flourished since the 1980s due to the discovery that the solutions of stochastic ordinary differential equations yield a cocycle over a metric dynamical system which models randomness, i.e. a random dynamical system. Arnold and I.D. Chueshov (1998) [2] presented the universal view of an order-preserving random dynamical system, offered several examples and studied the chattels of their random equilibria and attractor. Son (2009)[3] studied the Lyapunov exponents for random dynamical systems. Yingchao (2010)[4] used the theory of random dynamical systems and stochastic analysis to research the existence of random attractors and also stochastic bifurcation behavior for stochastic Duffing-van der Pol equation with jumps under some assumptions. Kadhim and A.H. Khalil(2016)[5] they define the random dynamical system and random sets in uniform space are and proved some necessary properties of these two concepts. Also they study the expansivity of uniform random operator.

The structure of this paper is as follows: In Section 2 we recall same basic definition and facts about random dynamical. In Section 3 we study the definition of trajectories in random dynamical system. In Section 4 we recall some basic fact about omega-limit random set in random dynamical system. In Section 5 will be devoted to the concept of prolongations and prolongational limit random sets under a random dynamical system. We define the first prolongations and prolongational limit random sets of random dynamical system (Definition 5.1,5.5) .If $M(\omega)$ is invariant. We have first prolongations and prolongational limit sets of random dynamical system so invariant ( Theorem5.3, 5.7 ). the first prolongation and the prolongational limit random set are closed sets (Theorem5.2,5.6) .If $X$ is locally compact. We have first prolongations and prolongational limit sets of random dynamical system are connected ( Theorem5.4, 4.13).

\section{Notation and basic definitions}

In this Section we recall some basic definition and facts about random dynamical system and notation .

\subsection{Notations}

(1) $\mathbb{G}=$ locally compact group

(2) $\mathbb{X}=$ metric space with metric $d$.

(3) $(\Omega, \mathbb{F}, \mathbb{P})$ is a probability space.
(4) $\mathbb{X}_{\mathcal{B}}^{\Omega}=$ the set of all measurable functions from $\Omega$ to X.

(5) $S[A, r] \quad$ the $\operatorname{set}\{y: d(y, A) \leq r\}$.

(6) $H(A, r) \quad$ the $\operatorname{set}\{y: d(y, A)=r\}$.

\subsection{Basic definitions}

Definition 2.2.1 [6-7]: The metric dynamical system (MDS) is the 5-tuple $(\mathbb{G}, \Omega, \mathbb{F}, \mathbb{P}, \theta)$ where $(\Omega, \mathbb{F}, \mathbb{P})$ is a probability space and $\theta: G \times \Omega \rightarrow \Omega$ is $(\beta(\mathbb{G}) \otimes$ F, F) - measurable, with

(i) $\quad \theta(e, \omega)=I d_{\Omega}$,

(ii) $\quad \theta(g * h, \omega)=\theta(g, \theta(h, \omega))$ and

(iii) $\quad \mathbb{P}\left(\theta_{g} F\right)=\mathbb{P}(F), \forall F \in \mathbb{F} \forall \omega \in G$.

Definition2.2.2[6]: The $\operatorname{MDS}(\mathbb{G}, \Omega, \mathbb{F}, \mathbb{P}, \theta)$ is said to be topological metric dynamical system (TMDS) if $\Omega$ is topological space and $\theta: G \times \Omega \rightarrow \Omega$ is continuous.

Definition2.2.3 [6-8]: The mapping $\varphi: \mathbb{G} \times \Omega \times \mathbb{X} \rightarrow$ $\mathbb{X}$ is said to be measurable random dynamical system on the measurable space $(\mathbb{X}, \beta(\mathbb{X}))$ over an $\operatorname{MDS}(\mathbb{G}, \Omega, \mathbb{F}, \mathbb{P}, \theta)$ with if it has the following properties:

(i) $\varphi$ is $\beta(\mathbb{G}) \otimes \mathbb{F} \otimes \beta(\mathbb{X}), \beta(\mathbb{X})-$ measurable.

(ii) The mappings $\varphi(t, \omega):=\varphi(g, \omega, \cdot): \mathbb{X} \rightarrow \mathbb{X}$ form a cocycle over $\theta(\cdot)$, that is, $\forall g, h \in \mathbb{G}, \omega \in \Omega$ they satisfy

$$
\varphi(e, \omega)=i d_{X} \forall \omega \in \Omega, \quad \text { (2.2.1) }
$$

$\varphi(g * h, \omega)=\varphi\left(g, \theta_{h} \omega\right) \circ \varphi(h, \omega)$

The RDS $(\mathbb{G}, \Omega, \mathbb{X}, \theta, \varphi)$ shall denote by $(\theta, \varphi)$.

If the function $\varphi(\cdot, \omega, \cdot): \mathbb{T} \times \mathbb{X} \rightarrow \mathbb{X}, \quad(t, x) \mapsto$ $\varphi(t, \omega, x)$, is continuous for every $\omega \in \Omega$ then the measurable dynamical system is called continuous or topological $\mathrm{R}$

Definition 2.2.4 [9]: Let $(\theta, \varphi)$ be a measurable RDS and $C \subset \Omega \times X$ a set.

(i) $C$ is called forward invariant if for $t>0$

$C(\omega) \subset \varphi(t, \omega)^{-1} C(\theta(t, \omega)) \mathbb{P}-$ a.s. equivalently

$$
\varphi(t, \omega) C(\omega) \subset C(\theta(t, \omega)) \mathbb{P}-\text { a.s.. }
$$

(ii) $C$ is called invariant if for all $t \in \mathbb{T}$

$$
C(\omega)=\varphi(t, \omega)^{-1} C(\theta(t, \omega)) \mathbb{P}-\text { a.s., }
$$

for two-sided time equivalent to

$$
\varphi(t, \omega) C(\omega)=C(\theta(t, \omega)) \mathbb{P}-\mathrm{a} .
$$

Definition 2.2.5 [9-10]: Let $(\Omega, \mathcal{F})$ be a measurable space and $(X, d)$ be a metric space which is considered a measurable space with Borel $\sigma-$ algebra $\mathcal{B}(\mathbb{X})$. The setvalued function $A: \Omega \rightarrow \mathcal{B}(\mathbb{X}), \omega \mapsto A(\omega)$, is said to be random set if for each $x \in \mathrm{X}$ the function $\omega \longmapsto$ $d(x, A(\omega))$ is measurable. If $A(\omega)$ is closed (connected) (compact) for all $\omega \in \Omega$, it is called a random closed (connected) (compact) set. 


\section{Definition 2.2.6 [10]:}

An $\operatorname{RDS}(\theta, \varphi)$ is said to be asymptotically compact in the universe $\mathcal{D}$, if there exists an attracting random compact set $\left\{B_{0}(\omega)\right\}$, i.e., foe any $D \in \mathcal{D}$ and for any $\omega \in \Omega$ we have

$\left.B_{0}(\omega)\right\}=0, \quad(2.2 .3)$

$$
\lim _{t \rightarrow \infty} d_{X}\{\varphi(t, \theta(-t) \omega) D(\theta(-t) \omega /
$$

where $d_{X}\{A / B\}=\sup _{x \in A} \operatorname{dist}(x, B)$.

\section{Definitions and characterizations}

In this section we study the trajectories in random dynamical system. First we shall state the definition of trajectories in random dynamical system and We describe some measurable properties of the trajectory of random dynamical system.

Definition 3.1: Let $\quad D: \omega \mapsto D(\omega)$ be a multifunction. We call the multifunction

$$
\omega \longmapsto \gamma_{D}^{t}(\omega):=\bigcup_{\tau \geq t} \varphi\left(\tau, \theta_{-\tau} \omega\right) D\left(\theta_{-\tau} \omega\right)
$$

the tail (from the moment $t$ ) of the pull back trajectories emanating from $D$. If $D(\omega)=\{v(\omega)\}$ is a single valued function, then $\omega \mapsto \gamma_{v}(\omega)=\gamma_{D}^{0}(\omega)$ is said to be the (pull back) trajectory ( or orbit) emanating from $v$. That is $\omega \mapsto \gamma_{v}(\omega):=$ $\bigcup_{\tau \geq 0} \varphi\left(\tau, \theta_{-\tau} \omega\right) v\left(\theta_{-\tau} \omega\right)$

Definition 3.2: Let $v \in \mathbb{X}_{\mathcal{B}_{\mathcal{B}}}^{\Omega}$ and $\gamma_{v}, \gamma_{v}^{+}$and $\gamma_{v}^{-}$be the mappings form $X$ in to $2^{X}$ defined as follows

(1) $\gamma_{v}(\omega)=\left\{\varphi\left(t, \theta_{-t} \omega\right) v\left(\theta_{-t} \omega\right): t \in \mathbb{R}\right\}$

(2) $\gamma_{v}^{+}(\omega)=\left\{\varphi\left(t, \theta_{-t} \omega\right) v\left(\theta_{-t} \omega\right): t \in \mathbb{R}^{+}\right\}$

(3) $\gamma_{v}^{-}(\omega)=\left\{\varphi\left(t, \theta_{-t} \omega\right) v\left(\theta_{-t} \omega\right): t \in \mathbb{R}^{-}\right\}$

For every $v \in X_{\mathcal{B}}^{\Omega}$, the sets $\gamma_{v}, \gamma_{v}^{+}$, and $\gamma_{v}^{-}$are respectively called the trajectory, the forward semitrajectory and backward semi-trajectory.

Definition 3.3: Let $x \in \mathbb{X}$. and $\gamma_{x}, \gamma_{x}^{+}$and $\gamma_{x}^{-}$be the mappings form $\mathbb{X}$. in to $2^{\mathbb{X}}$ defined as follows

(1) $\gamma_{x}(\omega)=\{\varphi(t, \omega) x: t \in \mathbb{R}\}$

(2) $\gamma_{x}^{+}(\omega)=\left\{\varphi(t, \omega) x: t \in \mathbb{R}^{+}\right\}$

(3) $\gamma_{x}^{-}(\omega)=\left\{\varphi(t, \omega) x: t \in \mathbb{R}^{-}\right\}$

For every $x \in X$, the sets $\gamma_{x}, \gamma_{x}^{+}$, and $\gamma_{x}^{-}$are respectively called the trajectory, the forward semi-trajectory and backward semi-trajectory.

Proposition 3.4: For and $v \in \mathbb{X}_{\cdot}^{\Omega}$, the sets $\gamma_{v}, \gamma_{v}^{+}$, and $\gamma_{v}^{-}$are invariant random sets.

Proof. Let $v \in \mathbb{X}_{\mathcal{B}}^{\Omega}$. To show that $\gamma_{v}$ is an invariant. Let $x \in \gamma_{v}(\omega)$ and $t \in \mathbb{R}$. Then there exists $s \in \mathbb{R}$ such that $x=\varphi\left(s, \theta_{-s} \omega\right) v\left(\theta_{-s} \omega\right)$. Now

$\mathbb{P}\left\{\omega: \varphi(t, \omega) x \in \gamma_{v}\left(\theta_{t} \omega\right)\right\}=$

$\mathbb{P}\left\{\omega: x \in \varphi\left(-t, \theta_{t} \omega\right) \gamma_{v}\left(\theta_{t} \omega\right)\right\}$

$\left.\varphi\left(-t, \theta_{t} \omega\right) \gamma_{v}\left(\theta_{t} \omega\right)\right\}$

$$
=\mathbb{P}\left\{\omega: \varphi\left(s, \theta_{-s} \omega\right) v\left(\theta_{-s} \omega\right) \in\right.
$$

$\left.\varphi\left(-t, \theta_{t} \omega\right) \gamma_{v}\left(\theta_{t} \omega\right)\right\}$

$$
=\mathbb{P}\left\{\omega: v\left(\theta_{-s} \omega\right) \in \varphi(-s, \omega) \circ\right.
$$

$\left.\varphi\left(-t, \theta_{t} \omega\right) \gamma_{v}\left(\theta_{t} \omega\right)\right\}$

$\left.\varphi\left(-t, \omega^{\prime}\right) \gamma_{v}\left(\omega^{\prime}\right)\right\}$

$$
=\mathbb{P}\left\{\omega: v\left(\theta_{-s} \omega\right) \in \varphi\left(-s, \theta_{-t} \omega^{\prime}\right) \circ\right.
$$

where $\omega^{\prime}=\theta_{t} \omega$.

$=\mathbb{P}\left\{\omega: v\left(\theta_{-s} \omega\right) \in \varphi\left(-s-t, \omega^{\prime}\right) \gamma_{v}\left(\omega^{\prime}\right)\right\}$

$$
\begin{array}{r}
=\mathbb{P}\left\{\theta_{-t} \omega^{\prime}: v\left(\theta_{-s} \theta_{-t} \omega^{\prime}\right) \in \varphi\left(-s-t, \omega^{\prime}\right) \gamma_{v}\left(\omega^{\prime}\right)\right\} \\
=\mathbb{P}\left\{\omega^{\prime}: v\left(\theta_{r} \omega^{\prime}\right) \in \varphi\left(r, \omega^{\prime}\right) \gamma_{v}\left(\omega^{\prime}\right)\right\},
\end{array}
$$$$
r=-s-t \text {. }
$$

$=\mathbb{P}\left\{\omega^{\prime}: \varphi\left(-r, \theta_{r} \omega^{\prime}\right) v\left(\theta_{r} \omega^{\prime}\right) \in \gamma_{v}\left(\omega^{\prime}\right)\right\}=1$.

Thus for every $x \in \gamma_{v}(\omega)$ and $t \in \mathbb{R}$, we have

$$
\mathbb{P}\left\{\omega: \varphi(t, \omega) x \in \gamma_{v}\left(\theta_{t} \omega\right)\right\}=1 \text {. }
$$

This means that the set $\gamma_{v}(\omega)$ is an invariant. In a similar way we can show that $\gamma_{v}^{+}$, and $\gamma_{v}^{-}$are invariant random sets.

\section{Omega-limit set in random dynamical system}

In this section, we state the definition of omega-limit set in random dynamical system is due to [10-11].Thus, we give some basic properties of omega-limit set in random dynamical system.

Definition 4.1: The multifunctions $\quad \omega \mapsto$ $\Gamma_{M}^{+}(\omega):=\{y \in \mathbb{X}$ :

there is a sequences $\left\{t_{n}\right\}$ in $\mathbb{R}$ and $\left\{x_{n}\right\}$ in $M\left(\theta_{-t_{n}} \omega\right)$ with $t_{n} \rightarrow$ $+\infty$ and $\varphi\left(t_{n}, \theta_{-t_{n}} \omega\right) x_{n} \rightarrow y$ for all $\left.\omega\right\}$

$\omega \longmapsto \Gamma_{M}^{-}(\omega):=\{y \in \mathbb{X} .:$

there is a sequence $\left\{t_{n}\right\}$ in $\mathbb{R}$ with $\left\{x_{n}\right\}$ in $M\left(\theta_{-t_{n}} \omega\right) t_{n} \rightarrow$ $-\infty$ and $\varphi\left(t_{n}, \theta_{-t_{n}}(\omega)\right) x_{n} \rightarrow y$ for all $\left.\omega\right\}$

are said to be the omega (alpha) -limit set of the trajectories emanating from $x$ respectively.

If $M=\{x\}$, the we have

『 $\omega \mapsto \Gamma \rrbracket \_x^{\wedge}+(\omega):=\{y \in \mathbb{X}$.

: there is a sequences $\left\{t_{-} n\right\}$ in $\mathbb{R}$ with $t_{-} n$

$\rightarrow+\infty$ and $\left.\varphi\left(t_{-} n, \theta_{-}(\mathbb{\zeta}-t]_{-} n\right) \omega\right) x \rightarrow y$ for all $\left.\omega\right\}$

$\omega \mapsto \Gamma_{x}^{-}(\omega):=\{y \in$

$\mathbb{X}$. : there is a sequence $\left\{t_{n}\right\}$ in $\mathbb{R}$ with $t_{n} \rightarrow$

$-\infty$ and $\varphi\left(t_{n}, \theta_{-t_{n}}(\omega)\right) x \rightarrow y$ for all $\left.\omega\right\}$.

The following assertion gives another description of omega-limit sets.

Theorem 4.2: Let $\Gamma_{M}^{+}(\omega)$ be the omega-limit set of the trajectories emanating from $M$. Then $\Gamma_{M}(\omega)=\bigcap_{t>0} \overline{\gamma_{D}^{t}(\omega)}=$ $\bigcap_{t>0} \overline{\mathrm{U}_{\tau \geq t} \varphi\left(\tau, \theta_{-\tau} \omega\right) M\left(\theta_{-\tau} \omega\right)}$

Proof. Suppose that $y \in \Gamma_{M}(\omega)$, the for any $t>0$ there exists $\left\{t_{n}\right\}$ in $\mathbb{R}$ and $\left\{x_{n}\right\}$ in $M\left(\theta_{-t_{n}} \omega\right)$ such that $\varphi\left(t_{n}, \theta_{-t_{n}} \omega\right) x_{n} \rightarrow y$. Hence $x_{n} \in$ $\mathrm{U}_{\tau \geq t} M\left(\theta_{-\tau} \omega\right)$. Thus

$\varphi\left(t_{n}, \theta_{-t_{n}} \omega\right) x_{n} \in \bigcup_{\tau \geq t} \varphi\left(\tau, \theta_{-\tau} \omega\right) M\left(\theta_{-\tau} \omega\right)$

Therefore

$$
\subset \overline{U_{\tau \geq t} \varphi\left(\tau, \theta_{-\tau} \omega\right) M\left(\theta_{-\tau} \omega\right)} \text {. }
$$

$$
y \in \overline{U_{\tau \geq t} \varphi\left(\tau, \theta_{-\tau} \omega\right) M\left(\theta_{-\tau} \omega\right)}, \text { for all } t>0 .
$$

Thus $y \in \bigcap_{t>0} \overline{\mathrm{U}_{\tau \geq t} \varphi\left(\tau, \theta_{-\tau} \omega\right) M\left(\theta_{-\tau} \omega\right)}$.

To prove the converse inclusion, let

$$
y \in \bigcap_{t>0} \overline{U_{\tau \geq t} \varphi\left(\tau, \theta_{-\tau} \omega\right) M\left(\theta_{-\tau} \omega\right)}
$$

then $y \in \overline{U_{\tau \geq t} \varphi\left(\tau, \theta_{-\tau} \omega\right) M\left(\theta_{-\tau} \omega\right)}$ for all $t>0$. In particular,

$1,2, \ldots$. 
Therefore there exists a sequence $\left\{y_{n}\right\}$ in $\bigcup_{\tau \geq n} \varphi\left(\tau, \theta_{-\tau} \omega\right) M\left(\theta_{-\tau} \omega\right)$ such that $y_{n} \rightarrow y$. Thus $y_{n} \in \bigcup_{\tau \geq n} \varphi\left(\tau, \theta_{-\tau} \omega\right) M\left(\theta_{-\tau} \omega\right)$ and $d\left(y, y_{n}\right)<$ $1 / n, n=1,2, \ldots$. It follows that there exists $t_{n} \geq n$ and $x_{n} \in M\left(\theta_{-\tau} \omega\right)$ such that $y_{n}=\varphi\left(t_{n}, \theta_{t_{n}} \omega\right) x_{n}$. That is $\varphi\left(t_{n}, \theta_{t_{n}} \omega\right) x_{n} \rightarrow y$. Consequently, $y \in \Gamma_{M}(\omega)$.

$\Gamma_{M}(\omega)=\bigcap_{t>0} \overline{\gamma_{D}^{t}(\omega)}=$

$\bigcap_{t>0} \overline{U_{\tau \geq t} \varphi\left(\tau, \theta_{-\tau} \omega\right) M\left(\theta_{-\tau} \omega\right)}$.

$\Gamma_{M}(\omega)=\bigcap_{t>0} \overline{\gamma_{D}^{t}(\omega)}=$

$\bigcap_{t>0} \overline{\mathrm{U}_{\tau \geq t} \varphi\left(\tau, \theta_{-\tau} \omega\right) M\left(\theta_{-\tau} \omega\right)}$. Since $\overline{\gamma_{D}^{t}(\omega)}$ is closed an invariant, then so is $\Gamma_{M}(\omega)=\bigcap_{t>0} \overline{\gamma_{D}^{t}(\omega)}$.

Theorem 4.3: Let $\Gamma_{M}^{+}(\omega)$ is a random closed set, then the proof is devided in two parts:

1: Indirect Proof. By above theorem we have

$\Gamma_{M}(\omega)=\bigcap_{t>0} \overline{\gamma_{D}^{t}(\omega)}=$

$\bigcap_{t>0} \overline{U_{\tau \geq t} \varphi\left(\tau, \theta_{-\tau} \omega\right) M\left(\theta_{-\tau} \omega\right)}$

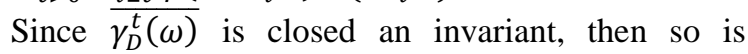
$\Gamma_{M}(\omega)=\bigcap_{t>0} \overline{\gamma_{D}^{t}(\omega)}$.

2: Direct proof. Let $y \in \overline{\Gamma_{M}^{+}(\omega)}$. Then there exists $\left\{y_{n}\right\}$ in be a sequence in $\Gamma_{M}^{+}(\omega)$ such that $y_{n} \rightarrow y$. We wish to show that $y \in \Gamma_{M}^{+}(\omega)$. Indeed for each positive integer $k$, there is a sequence $\left\{t_{n}^{k}\right\}$ in $\mathbb{R}$ and $\left\{x_{n}^{k}\right\} \quad$ in $M\left(\theta_{-t_{n}^{k}} \omega\right) \quad$ with $t_{n}^{k} \longrightarrow+\infty$ and $\varphi\left(t_{n}^{k}, \theta_{-t_{n}^{k}}(\omega)\right) x_{n}^{k} \rightarrow y_{k}$. We assume without loss of generality that $d\left(y_{k}, \varphi\left(t_{n}^{k}, \theta_{-t_{n}^{k}} \omega\right) x_{n}^{k}\right)<1 / k$ and $t_{n}^{k} \geq k$ for $n \geq k$. Consider now the sequence $\left\{t_{n}\right\}$ in $\mathbb{R}$ with $t_{n}=t_{n}^{n}$ and a sequence $\left\{x_{n}\right\}$ in $M\left(\theta_{-t_{n}} \omega\right)$ with

$x_{n}=x_{n}^{n}$. Then $t_{n} \rightarrow+\infty$ and we claim that $\varphi\left(t_{n}^{k}, \theta_{-t_{n}^{k}} \omega\right) x_{n} \rightarrow y$.To see this observe that $d\left(\varphi\left(t_{n}, \theta_{-t_{n}} \omega\right) x_{n}, y\right) \leq d\left(\varphi\left(t_{n}, \theta_{-t_{n}} \omega\right) x_{n}, y_{n}\right)+$ $d\left(y_{n}, y\right)$

$$
<1 / n+d\left(y_{n}, y\right) \text {. }
$$

Since $1 / n$ and $d\left(y_{n}, y\right)$ tend to zero we conclude that

$$
d\left(\varphi\left(t_{n}, \theta_{-t_{n}} \omega\right) x_{n}, y\right) \rightarrow 0
$$

Consequently $\varphi\left(t_{n}, \theta_{-t_{n}} \omega\right) x_{n} \rightarrow y$ and $y \in$ $\Gamma_{M}^{+}(\omega)$. Thus $\Gamma_{M}^{+}(\omega)=\overline{\Gamma_{M}^{+}(\omega)}$, i.e., $\Gamma_{M}^{+}(\omega)$ is closed. Theorem 4.4: Let $\mathbb{X}$. be any metric space and $x \in \mathbb{X}$. . Then

$$
\Gamma_{x}^{+}\left(\theta_{t} w\right)=\varphi(t, \omega) \Gamma_{x}^{+}(\omega) \text { for every } t \in \mathbb{R} \text {. }
$$

Proof. To prove $\Gamma_{x}^{+}\left(\theta_{t} w\right)=\varphi(t, \omega) \Gamma_{x}^{+}(\omega)$.Let $\epsilon$ $\Gamma_{x}^{+}\left(\theta_{t} w\right)$.Then there is a sequences $\left\{t_{n}\right\}$ in $\mathbb{R}$ with $t_{n} \rightarrow$ $+\infty$ and $\varphi\left(t_{n}, \theta_{-t_{n}} \theta_{t} w\right) x \rightarrow z$

$$
\begin{gathered}
\varphi\left(t_{n}+t-t, \theta_{-t_{n}} \theta_{t} w\right) x \rightarrow z \\
\varphi(t, \omega) \varphi\left(t_{n}-t, \theta_{-t_{n}} \theta_{t} w\right) x \rightarrow z
\end{gathered}
$$

$\varphi\left(t_{n}-t, \theta_{t-t_{n}} w\right) x \rightarrow \varphi(t, \omega)^{-1} z \quad$,where $t_{n}-t \rightarrow+\infty$. Thus we have $\varphi(t, \omega)^{-1} z \in \Gamma_{x}^{+}(\omega)$. Then $z \in \varphi(t, \omega) \Gamma_{x}^{+}(\omega)$

Then $\Gamma_{x}^{+}\left(\theta_{t} w\right) \subseteq \varphi(t, \omega) \Gamma_{x}^{+}(\omega)$.Now let $z \in$ $\varphi(t, \omega) \Gamma_{x}^{+}(\omega)$. Then is $y \in \Gamma_{x}^{+}(\omega)$ suchthat $\quad z=\varphi(t, \omega) y$.Then there is a sequences $\left\{t_{n}\right\}$ in $\mathbb{R}$ with $t_{n} \rightarrow$ $+\infty$ and $\varphi\left(t_{n}, \theta_{-t_{n}}\right) x \rightarrow y$. By continuity $\varphi(t, \omega)$, $\varphi(t, \omega) \varphi\left(t_{n}, \theta_{-t_{n}} w\right) x \rightarrow \varphi(t, \omega) y \quad, \varphi(t+$ $\left.t_{n}, \theta_{-t_{n}} w\right) x \rightarrow z$

$\varphi\left(t_{n}+t, \theta_{-t-t_{n}} \theta_{t} w\right) x \rightarrow z \quad$.Thus we have $z \in \Gamma_{x}^{+}\left(\theta_{t} w\right)$.Then

$\varphi(t, \omega) \Gamma_{x}^{+}(\omega) \subseteq \Gamma_{x}^{+}\left(\theta_{t} w\right) \quad$.Then $\Gamma_{x}^{+}\left(\theta_{t} w\right)=$ $\varphi(t, \omega) \Gamma_{x}^{+}(\omega)$.

Theorem 4.5.If $\quad(t, x) \mapsto \varphi\left(t, \theta_{-t} \omega\right) x \quad$ is continuous, then

$$
\overline{\gamma_{M}^{t}(\omega)}=\gamma_{M}^{t}(\omega) \cup \Gamma_{M}^{+}(\omega) \text {. }
$$

Proof. First, note that $\gamma_{M}^{t}(\omega) \subset \overline{\gamma_{M}^{t}(\omega)}$. By Theorem (4.2), we have $\quad \Gamma_{M}^{+}(\omega) \subset \overline{\gamma_{M}^{t}(\omega)}$. Therefore $\overline{\gamma_{M}^{t}(\omega)} \supset \gamma_{M}^{t}(\omega) \cup \Gamma_{M}^{+}(\omega)$. To prove the converse inclusion, let $y \in \overline{\gamma_{M}^{t}(\omega)}$. then there exists a sequence $\left\{y_{n}\right\}$ in $\gamma_{M}^{t}(\omega)$ such that $y_{n} \rightarrow y$. Now $y_{n} \in \bigcup_{\tau \geq t} \varphi\left(\tau, \theta_{-\tau} \omega\right) M\left(\theta_{-\tau} \omega\right)$, then there exists a sequence $\left\{\tau_{n}\right\}$ with $\tau_{n} \geq t$ for every $n$ and $\left\{x_{n}\right\}$ in $M\left(\theta_{-\tau_{n}} \omega\right)$ such that $y_{n}=\varphi\left(\tau_{n}, \theta_{-\tau_{n}} \omega\right) x_{n}$. We have two cases:

Case I: The sequence $\left\{\tau_{n}\right\}$ has the property that $\tau_{n} \rightarrow+\infty$, in which case $y \in \Gamma_{M}^{+}(\omega)$.

Case II: There is a subsequence $\left\{\tau_{n_{k}}\right\}$ in $\mathbb{R}^{+}$such that $\tau_{n_{k}} \rightarrow \tau \in \mathbb{R}^{+}$(as $\mathbb{R}^{+}$is closed). But then $\varphi\left(\tau_{n_{k}}, \theta_{-\tau_{n_{k}}} \omega\right) x \rightarrow \varphi\left(\tau, \theta_{-\tau} \omega\right) x \in \gamma_{M}^{t}(\omega)$ (since $(t, x) \mapsto \varphi\left(t, \theta_{-t} \omega\right)$ (since $(t, x) \mapsto \varphi\left(t, \theta_{-t} \omega\right) x$ is continuous). Since $\left(\tau_{n_{k}}, \theta_{-\tau_{n_{k}}} \omega\right) x \rightarrow y$, then from the uniqueness of the limit we have $\varphi\left(\tau, \theta_{-\tau} \omega\right) x=$ $y \in \gamma_{M}^{t}(\omega)$. From Case I and Case II, we have $y \in \gamma_{M}^{t}(\omega) \cup \Gamma_{M}^{+}(\omega)$. Hence

$$
\overline{\gamma_{M}^{t}(\omega)} \subset \gamma_{M}^{t}(\omega) \cup \Gamma_{M}^{+}(\omega) \text {. }
$$

Therefore $\overline{\gamma_{M}^{t}(\omega)}=\gamma_{M}^{t}(\omega) \cup \Gamma_{M}^{+}(\omega)$

Corollary 4.6: For any $x \in \mathbb{X} . \overline{\gamma_{x}^{+}(\omega)}=\gamma_{x}^{+}(\omega) \cup$ $\Gamma_{x}^{+}(\omega)$ and $\overline{\gamma_{x}^{-}(\omega)}=\gamma_{x}^{-}(\omega) \cup \Gamma_{x}^{-}(\omega)$.

Proof. By the definition we have $\gamma_{x}^{+}(\omega) \cup$ $\Gamma_{x}^{+}(\omega) \subseteq \overline{\gamma_{x}^{+}(\omega)}$. To show that $\overline{\gamma_{x}^{+}(\omega)} \subseteq \gamma_{x}^{+}(\omega) \cup$ $\Gamma_{x}^{+}(\omega)$,let $y \in \overline{\gamma_{x}^{+}(\omega)}$. Then there is a sequence $\left\{y_{n}\right\}$ $\operatorname{in} \gamma_{x}^{+}(\omega)$ such that $y_{n} \rightarrow y$. Since $y_{n}$ in $\gamma_{x}^{+}(\omega)$.Then $y_{n}=\varphi\left(\tau_{n}, \theta_{-\tau_{n}} \omega\right) x$ for a $\tau_{n}$ in $R^{+}$.Either the sequence $\left\{\tau_{n}\right\}$ has the property that $\tau_{n} \rightarrow+\infty$,in which case $y \in \Gamma_{x}^{+}(\omega)$, or there is a subsequence $\tau_{n k} \rightarrow t \in R^{+}$(as $R^{+}$is closed).But then $\varphi\left(\tau_{n k}, \theta_{-\tau_{n}} \omega\right) x \rightarrow \varphi\left(\tau, \theta_{-\tau} \omega\right) x \in \gamma_{x}^{+}(\omega)$, and since also $\varphi\left(\tau_{n k}, \theta_{-\tau_{n}} \omega\right) x \rightarrow y \quad$ we $\operatorname{have} \varphi\left(\tau, \theta_{-\tau} \omega\right) x=y \in \gamma_{x}^{+}(\omega)$. Thus $\overline{\gamma_{x}^{+}(\omega)} \subseteq$ $\gamma_{x}^{+}(\omega) \cup \Gamma_{x}^{+}(\omega)$. Thus $\overline{\gamma_{x}^{+}(\omega)}=\gamma_{x}^{+}(\omega) \cup \Gamma_{x}^{+}(\omega)$ 


\section{Some Properties of the Limit Random Sets in Random Dynamical Systems.}

the concepts of prolongations and prolongational limit sets are played an essential role. In the deterministic dynamical system the formal definition of prolongation is due to Ura [12] and the concept of prolongational limit set is due to Bhatia, Szegö [13]. By following this line of investigation, the present paper introduces the notions of prolongations and prolongational limit random sets of random dynamical systems. We simplify several concepts and effects of reclusiveness and depressiveness from Bhatia and Szegö [2]. We consider $(\theta, \varphi)$ random dynamical system then we define the first prolongations and prolongational limit random set of $M$.we prove some new properties of the studying of prolongations and prolongational limit random sets.

Definition 5.1: Let $M: \omega \mapsto M(\omega)$ be multifunction. The multifunction $\omega \mapsto D_{M}^{+}(\omega)$, where

$D_{M}^{+}(\omega):=\{y \in \mathbb{X}$.

: there is a sequences $\left\{t_{n}\right\}$ in $\mathbb{R}^{+}$and $\left\{x_{n}\right\} \operatorname{in} M\left(\theta_{-t_{n}} \omega\right)$ with , $x_{n} \rightarrow x \in$

$\bigcap_{n=1}^{\infty} M\left(\theta_{-t_{n}} \omega\right)$ and $\varphi\left(t_{n}, \theta_{-t_{n}} \omega\right) x_{n} \rightarrow y$ for all $\left.\omega\right\}$, is said to be to be first positive prolongation of $M$. If the set $\mathbb{R}^{+}$replaced by $\mathbb{R}^{-}$in above we get the notation of first negative prolongation of $M$ and shall denoted by $D_{M}^{-}(\omega)$.

If $M=\{x\}$, the we have

$D_{x}^{+}(\omega):=\{y \in \mathbb{X}$ : :

there is a sequences $\left\{t_{n}\right\}$ in $\mathbb{R}^{+}$and $\left\{x_{n}\right\}$ in $X$ with , $x_{n}$ $x$ and $\varphi\left(t_{n}, \theta_{-t_{n}} \omega\right) x_{n} \rightarrow y$ for all $\left.\omega\right\}$

Theorem 5.2: $D_{M}^{+}(\omega)$ is closed.

Proof. To show that $D_{M}^{+}(\omega)$ is closed. Let $y \in \overline{\mathrm{D}_{M}^{+}(\omega)}$ then there exists sequence $\left\{y_{n}\right\}$ in $\mathrm{D}_{M}^{+}(\omega)$ such that $y_{n} \rightarrow y$. Since $y_{n} \in \mathrm{D}_{M}^{+}(\omega)$ for every $n$. Then by definition of $\mathrm{D}_{M}^{+}(\omega)$ there exists sequences $\left\{t_{n}^{k}\right\} \in \mathbb{R}^{+}$ and $\quad\left\{x_{n}^{k}\right\} \in M\left(\theta_{-t_{n}^{k}} \omega\right)$ such that $x_{n}^{k} \rightarrow x \in$ $\cap_{n=1}^{\infty} M\left(\theta_{-t_{n}^{k}} \omega\right) \quad$ and $\quad \varphi\left(t_{n}^{k}, \theta_{-t_{n}^{k}} \omega\right) x_{n}^{k} \rightarrow y_{k}$. We assume by taking subsequences if necessarily that $t_{n}^{k}>k, d\left(x_{n}^{k}, x\right) \leq 1 / k$ and $d\left(\varphi\left(t_{n}^{k}, \theta_{-t_{n}^{k}} \omega\right) x_{n}^{k}, y_{k}\right) \leq$ $1 / k$ for $n \geq k$. Now consider the sequences $\left\{x_{n}^{n}\right\},\left\{t_{n}^{n}\right\}$. Clearly $x_{n}^{n} \rightarrow x \in \bigcap_{n=1}^{\infty} M\left(\theta_{\left.-t_{n}^{n} \omega\right)}\right.$ and $\left\{t_{n}^{n}\right\} \in \mathbb{R}^{+}$. Note that

$$
\begin{aligned}
& d\left(\varphi\left(t_{n}^{n}, \theta_{-t_{n}^{n}} \omega\right) x_{n}^{n}, y\right) \leq d\left(\varphi\left(t_{n}^{n}, \theta_{-t_{n}^{n}} \omega\right) x_{n}^{n}, y_{n}\right)+ \\
& d\left(y_{n}, y\right)
\end{aligned}
$$$$
\leq 1 / n+d\left(y_{n}, y\right) \text {. }
$$

Since $\{1 / n\}$ and $d\left(y_{n}, y\right)$ tend to zero, then $\varphi\left(t_{n}^{n}, \theta_{-} t_{n}^{n} \omega\right) x_{n}^{n} \rightarrow y$, then $y \in D_{M}^{+}(\omega)$. This means $\overline{\mathrm{D}_{M}^{+}(\omega)}=\mathrm{D}_{M}^{+}(\omega)$ and so $\mathrm{D}_{M}^{+}(\omega)$ is closed.

Theorem 4.3: If $M$ is invariant, then so is $\mathrm{D}_{M}^{+}(\omega)$. Proof. We need to show that $\varphi(t, \omega) \mathrm{D}_{M}^{+}(\omega)=$ $\mathrm{D}_{M}^{+}\left(\theta_{t} \omega\right)$.
Let $z \in \varphi(t, \omega) \mathrm{D}_{M}^{+}(\omega)$, then there exists $y \in \mathrm{D}_{M}^{+}(\omega)$ such that

$$
z=\varphi(t, \omega) y .
$$

To show that $z \in \mathrm{D}_{M}^{+}\left(\theta_{t} \omega\right)$. Since $y \in \mathrm{D}_{M}^{+}(\omega)$, there exist sequences $\left\{t_{n}\right\}$ in $\mathbb{R}^{+}$and $\left\{x_{n}\right\}$ in $M\left(\theta_{-t_{n}} \theta_{t} \omega\right)$ with $x_{n} \rightarrow x \in \bigcap_{n=1}^{\infty} M\left(\theta_{-t_{n}} \omega\right)$ and

$\varphi\left(t_{n}, \theta_{-t_{n}} \omega\right) x_{n} \rightarrow y$. Since $\varphi(t, \omega)$ is continuous, then

$$
\begin{aligned}
& \varphi(t, \omega) \circ \varphi\left(t_{n}, \theta_{-t_{n}} \omega\right) x_{n} \rightarrow \varphi(t, \omega) y, \text { then } \\
& \varphi\left(t+t_{n}, \theta_{-t-t_{n}} \circ \theta_{t} \omega\right) x_{n} \rightarrow \varphi(t, \omega) y
\end{aligned}
$$

for and $y \in \mathrm{D}_{M}^{+}(\omega)$ by Definition. According to Def. $z=\varphi(t, \omega) y \in \mathrm{D}_{M}^{+}\left(\theta_{t} \omega\right)$. then $\varphi(t, \omega) \mathrm{D}_{M}^{+}(\omega) \subset$ $\mathrm{D}_{M}^{+}\left(\theta_{t} \omega\right)$. To prove the converse inclusion, let $z \in$ $\mathrm{D}_{M}^{+}\left(\theta_{t} \omega\right)$. by Def. there exist sequences $\left\{t_{n}\right\}$ in $\mathbb{R}^{+}$and $\left\{y_{n}\right\}$ in $M\left(\theta_{-t_{n}} \theta_{t} \omega\right)$ with

$y_{n} \rightarrow y \in \bigcap_{n=1}^{\infty} M\left(\theta_{-t_{n}} \theta_{t} \omega\right)$ and so

$y \in M\left(\theta_{-t_{n}} \theta_{t} \omega\right)$ for all $n$. Since $M$ is an invariant $y_{n} \in M\left(\theta_{-t_{n}} \theta_{t} \omega\right)$, then

$y_{n} \in \varphi\left(t, \theta_{-t_{n}} \omega\right) M\left(\theta_{-t_{n}} \omega\right)$, then there exists $x_{n} \in$ $M\left(\theta_{-t_{n}} \omega\right)$ such that $y_{n}=\varphi\left(t, \theta_{-t_{n}} \omega\right) x_{n}$, then $x_{n}=\varphi\left(-t, \theta_{t} \theta_{-t_{n}} \omega\right) y_{n}$. Now,

$$
y \in \bigcap_{n=1}^{\infty} \varphi\left(t, \theta_{-t_{n}} \omega\right) M\left(\theta_{-t_{n}} \omega\right) .
$$

Then $y \in \varphi\left(t, \theta_{-t_{n}} \omega\right) M\left(\theta_{-t_{n}} \omega\right)$ for all $n$.

Then there exists $x \in \cap_{n=1}^{\infty} M\left(\theta_{-t_{n}} \omega\right)$ such that $y=\varphi\left(t, \theta_{-t_{n}} \omega\right) x$ for all $n$. Since $y_{n} \rightarrow y$, i.e. $\varphi\left(t_{n}, \theta_{-t_{n}} \omega\right) x_{n} \rightarrow \varphi\left(t, \theta_{-t_{n}} \omega\right) x$

$$
\begin{aligned}
& \varphi\left(t_{n}, \theta_{-t_{n}} \theta_{t} \omega\right) y_{n} \rightarrow z . \\
& \quad \varphi(t, \omega) \circ \varphi\left(t_{n}-t, \theta_{-t_{n}+t} \omega\right) x_{n} \rightarrow z
\end{aligned}
$$$$
\Rightarrow \varphi(t, \omega) z_{n} \rightarrow z, \text { with }
$$

$\rightarrow z_{n}:=\varphi\left(t_{n}-t, \theta_{-t_{n}+t} \omega\right) x_{n}$.

From (2.2.3) we have that $z_{n} \rightarrow B_{0}(\omega)$ as $n \rightarrow \infty$. Since $B_{0}(\omega)$ is compact, there exist $\left\{n_{k}\right\}$ and $b \in B_{0}(\omega)$ such that $z_{n_{k}} \rightarrow b$ as $k \rightarrow \infty$. Moreover by Def. $b \in \mathrm{D}_{M}^{+}(\omega)$. From (5.1) we obtain that $z=\varphi(t, \omega) b$. Therefore $\mathrm{D}_{M}^{+}\left(\theta_{t} \omega\right) \subset \varphi(t, \omega) \mathrm{D}_{M}^{+}(\omega)$ for all $t>0$ and $\omega \in \Omega$. Thus $\mathrm{D}_{M}^{+}(\omega)$ is invariant

We now discuss about the connectedness of the First Prolongation .

Theorem 4.4: Let $\mathbb{X}$. be locally compact. Then $\mathrm{D}_{M}^{+}(\omega)$ is connected whenever it is compact.

Proof. Let $\mathrm{D}_{M}^{+}(\omega)$ be compact but disconnected. Then there are two compact non- empty sets $P$ and $Q$ such that $P \cup Q=\mathrm{D}_{M}^{+}(\omega)$ and $P \cap Q=\varnothing$. Since $P$ and $Q$ are compact $d(P, Q)>0$.Thus there is $r>0$ such that $S[P, r], S[Q, r]$ are compact and disjoint .Now $x \in \mathrm{P}$ or $x \in \mathrm{Q}$.Let $x \in \mathrm{P}$. Then there is a sequence $\left\{x_{n}\right\}$ in $X$ and a sequence $\left\{t_{n}\right\}$ in $\mathbb{R}^{+}$such that $x_{n} \rightarrow x$, and $\varphi\left(t_{n}, \theta_{-t_{n}} \omega\right) x_{n} \rightarrow y \in Q$.We may assume $x_{n} \in S[P, r]$ and $\varphi\left(t_{n}, \theta_{-t_{n}} \omega\right) x_{n} \in S[Q, r]$.Then the trajectory segments $\varphi\left(s_{n}, \theta_{-s_{n}} \omega\right) x_{n} \quad, 0 \leq s_{n} \leq t_{n} \quad$ intersect $H(P, r)$,and therefor is a sequence $\left\{\tau_{n}\right\},, 0 \leq \tau_{n} \leq t_{n}$ such that $\varphi\left(\tau_{n}, \theta_{\tau_{n}} \omega\right) x_{n} \in H(P, r)$.Since $H(P, r)$ is 
compact we may assume that $\varphi\left(\tau_{n}, \theta_{\tau_{n}} \omega\right) x_{n} \rightarrow z \in$ $H(P, r)$. Then $\quad z \in \mathrm{D}_{M}^{+}(\omega)$, but $\quad z \notin \mathrm{P} \cup Q \quad$ as $\quad z \in$ $H(P, r)$. Thus contradiction shows that $\mathrm{D}_{M}^{+}(\omega)$ is connected.

Definition 5.5: Let $\quad M: \omega \mapsto M(\omega)$ be multifunction. The multifunction $\omega \longmapsto J_{M}^{+}(\omega)$, where $J_{M}^{+}(\omega):=\{y \in \mathbb{X}$.

: there is a sequences $\left\{t_{n}\right\}$ in $\mathbb{R}^{+}$and $\left\{x_{n}\right\}$ in $M\left(\theta_{-t_{n}} \omega\right)$ with $t_{n} \rightarrow+\infty, x_{n} \rightarrow M(\omega)$ and $\varphi\left(t_{n}, \theta_{-t_{n}} \omega\right) x_{n} \rightarrow$ $y$ for all $\omega\}$, is said to be to be first positive prolongational limit set of $M$. If the set $\mathbb{R}^{+}$replaced by $\mathbb{R}^{-}$in above we get the notation of first negative prolongational limit set of $M$ and shall denoted by $J_{M}^{-}(\omega)$.

If $M=\{x\}$, then the definition of $J_{M}^{+}(\omega)$ becomes $J_{x}^{+}(\omega):=\{y \in \mathbb{X}$.

: there is a sequences $\left\{t_{n}\right\}$ in $\mathbb{R}^{+}$and $\left\{x_{n}\right\}$ in $X$ with $t_{n} \rightarrow+\infty$,

$x_{n} \rightarrow x$ and $\varphi\left(t_{n}, \theta_{-t_{n}} \omega\right) x_{n} \rightarrow y$ for all $\left.\omega\right\}$.

The following result show that the prolongational limit set is closed and invariant.

Theorem 5.6: $J_{M}^{+}(\omega)$ is closed.

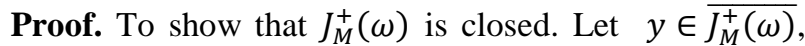
then there exists sequence $\left\{y_{n}\right\}$ in $J_{M}^{+}(\omega)$ such that $y_{n} \rightarrow y$. Since $y_{n} \in J_{M}^{+}(\omega)$ for every $n$. Then by definition of $J_{M}^{+}(\omega)$ there exists sequences $\left\{t_{n}^{k}\right\} \in \mathbb{R}^{+}$ and $\left\{x_{n}^{k}\right\} \in M\left(\theta_{-t_{n}^{k}} \omega\right)$ such that $x_{n}^{k} \rightarrow M(\omega)$, $t_{n}^{k} \rightarrow+\infty$ and $\varphi\left(t_{n}^{k}, \theta_{-t_{n}^{k}} \omega\right) x_{n}^{k} \rightarrow y_{k}$. We assume by taking subsequences if necessarily that $t_{n}^{k}>k$, $d\left(x_{n}^{k}, x\right) \leq 1 / k$ and $d\left(\varphi\left(t_{n}^{k}, \theta_{-t_{n}^{k}} \omega\right) x_{n}^{k}, y_{k}\right) \leq 1 / k$ for $n \geq k$. Now consider the sequences $\left\{x_{n}^{n}\right\},\left\{t_{n}^{n}\right\}$. Clearly $x_{n}^{n} \rightarrow M(\omega)$ and $t_{n}^{k} \rightarrow+\infty$. Note that

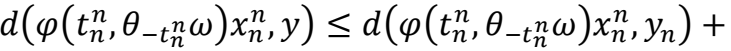
$d\left(y_{n}, y\right)$

$$
\leq 1 / n+d\left(y_{n}, y\right) \text {. }
$$

Since $\{1 / n\}$ and $d\left(y_{n}, y\right)$ tend to zero, then $\varphi\left(t_{n}^{n}, \theta_{-t_{n}^{n}} \omega\right) x_{n}^{n} \rightarrow y$, then $y \in J_{M}^{+}(\omega)$. This means $\overline{J_{M}^{+}(\omega)}=J_{M}^{+}(\omega)$ and so $J_{M}^{+}(\omega)$ is closed.

Theorem 4.7: If $M$ is invariant, then so is $J_{M}^{+}(\omega)$. Proof. We need to show that $\varphi(t, \omega) J_{M}^{+}(\omega)=$ $J_{M}^{+}\left(\theta_{t} \omega\right)$.

Let $z \in \varphi(t, \omega) J_{M}^{+}(\omega)$, then there exists $y \in J_{M}^{+}(\omega)$ such that $z=\varphi(t, \omega) y$.

To show that $z \in J_{M}^{+}\left(\theta_{t} \omega\right)$. Since $y \in J_{M}^{+}(\omega)$, there exist sequences $\left\{t_{n}\right\}$ in $\mathbb{R}^{+}$and $\left\{x_{n}\right\}$ in $M\left(\theta_{-t_{n}} \theta_{t} \omega\right)$ with $x_{n} \rightarrow M(\omega), t_{n} \rightarrow+\infty$ and $\varphi\left(t_{n}, \theta_{-t_{n}} \omega\right) x_{n} \rightarrow y$.

Since $\varphi(t, \omega)$ is continuous, then

$$
\varphi(t, \omega) \circ \varphi\left(t_{n}, \theta_{-t_{n}} \omega\right) x_{n} \rightarrow \varphi(t, \omega) y .
$$

By the cocycle property, we have

$$
\varphi\left(t+t_{n}, \theta_{-t-t_{n}} \circ \theta_{t} \omega\right) x_{n} \rightarrow \varphi(t, \omega) y
$$

for and $y \in J_{M}^{+}(\omega)$ by definition. According to definition. $z=\varphi(t, \omega) y \in J_{M}^{+}\left(\theta_{t} \omega\right)$. then $J_{M}^{+}(\omega) \subset$ $J_{M}^{+}\left(\theta_{t} \omega\right)$. To prove the converse inclusion, let $z \in$ $J_{M}^{+}\left(\theta_{t} \omega\right)$. By definition there exist sequences $\left\{t_{n}\right\}$ in $\mathbb{R}^{+}$ and $\left\{x_{n}\right\}$ in $M\left(\theta_{-t_{n}} \theta_{t} \omega\right)$ with $x_{n} \rightarrow M(\omega), t_{n} \rightarrow+\infty$ and $\varphi\left(t_{n}, \theta_{-t_{n}} \theta_{t} \omega\right) x_{n} \rightarrow z$. By the cocycle property we have

$$
\varphi(t, \omega) \circ \varphi\left(t_{n}-t, \theta_{-t_{n}+t} \omega\right) x_{n} \rightarrow z
$$

$\Rightarrow \varphi(t, \omega) z_{n} \rightarrow z$, with

$z_{n}:=\varphi\left(t_{n}-t, \theta_{-t_{n}+t} \omega\right) x_{n}$

From (2.2.3) we have that $z_{n} \rightarrow B_{0}(\omega)$ as $n \rightarrow \infty$. Since $B_{0}(\omega)$ is compact, there exist $\left\{n_{k}\right\}$ and $b \in B_{0}(\omega)$ such that $z_{n_{k}} \rightarrow b$ as $k \rightarrow \infty$. Moreover by Def. $b \in J_{M}^{+}(\omega)$. From (5.2) we obtain that $z=\varphi(t, \omega) b$. Therefore $J_{M}^{+}\left(\theta_{t} \omega\right) \subset \varphi(t, \omega) J_{M}^{+}(\omega)$ for all $t>0$ and $\omega \in \Omega$. Thus $J_{M}^{+}(\omega)$ is invariant.

Theorem 5.8: $\mathrm{D}_{M}^{+}(\omega)=\gamma_{M}^{+}(\omega) \cup J_{M}^{+}(\omega)$.

Proof. $\gamma_{M}^{+}(\omega) \cup J_{M}^{+}(\omega) \subset \mathrm{D}_{M}^{+}(\omega)$. To prove the converse inclusion. Let $y \in \mathrm{D}_{M}^{+}(\omega)$ by Def. there exist sequences $\left\{t_{n}\right\}$ in $\mathbb{R}^{+}$and $\left\{x_{n}\right\}$ in $M\left(\theta_{-t_{n}} \omega\right)$ with $x_{n} \rightarrow M(\omega)$ and $\varphi\left(t_{n}, \theta_{-t_{n}} \omega\right) x_{n} \rightarrow y$. We may assume that either $t_{n} \rightarrow t \in \mathbb{R}^{+}$or $t_{n} \rightarrow+\infty$, if necessarily by taking subsequences. In the first case $\varphi\left(t_{n}, \theta_{-t_{n}} \omega\right) x_{n} \rightarrow \varphi\left(t, \theta_{-t} \omega\right) x \quad$ (since $\varphi(\cdot, \omega, \cdot): \mathbb{R} \times$ $X \rightarrow X$ is continuous for every $\omega \in \Omega$ ). By uniqueness of the limit we have $\varphi\left(t, \theta_{-t} \omega\right) x=y \in \gamma_{M}^{+t}(\omega)$. In the second case $y \in J_{M}^{+}(\omega)$ by Def. of $J_{M}^{+}(\omega)$. Thus $y \in$ $\gamma_{M}^{+t}(\omega) \cup J_{M}^{+}(\omega)$. Hence $\mathrm{D}_{M}^{+}(\omega)=\gamma_{M}^{+}(\omega) \cup J_{M}^{+}(\omega)$.

Corollary 5.9: $D_{x}^{+}(\omega)=\gamma_{x}^{+}(\omega) \cup J_{x}^{+}(\omega)$.

Proof. By definitions $\gamma_{x}^{+}(\omega) \cup J_{x}^{+}(\omega) \subset \mathrm{D}_{x}^{+}(\omega)$. . To prove the converse inclusion. Let $y \in \mathrm{D}_{x}^{+}(\omega)$ by Def. there exist a sequences $\left\{t_{n}\right\}$ in $\mathbb{R}^{+}$and a sequences $\left\{x_{n}\right\}$ with $x_{n} \rightarrow x$ such that $\varphi\left(t_{n}, \theta_{-t_{n}} \omega\right) x_{n} \rightarrow y$. We may assume that either $t_{n} \rightarrow t \in \mathbb{R}^{+}$or $t_{n} \rightarrow+\infty$, if necessarily by taking subsequences. In the first case $\varphi\left(t_{n}, \theta_{-t_{n}} \omega\right) x_{n} \rightarrow \varphi\left(t, \theta_{-t} \omega\right) x \quad$ (since $\varphi(\cdot, \omega, \cdot): \mathbb{R} \times$ $X \rightarrow X$ is continuous for every $\omega \in \Omega$ ). By uniqueness of the limit we have $\varphi\left(t, \theta_{-t} \omega\right) x=y \in \gamma_{x}^{+}(\omega)$. In the second case $y \in J_{x}^{+}(\omega)$ by Def. of $J_{x}^{+}(\omega)$. Thus $y \in$ $\gamma_{x}^{+}(\omega) \cup J_{x}^{+}(\omega)$. Hence $\mathrm{D}_{x}^{+}(\omega)=\gamma_{x}^{+}(\omega) \cup J_{x}^{+}(\omega)$.

\section{Theorem5.10:Let}

$x, y \in X$ with the property that

$$
x=\varphi\left(t, \theta_{-t} \omega\right) y, \text { for every } t \text { in } \mathbb{R} \text { and }
$$

$\omega \in \Omega$. Then $y \in J_{x}^{+}(\omega)$ if and only if

$x \in J_{y}^{-}(\omega)$.

Proof. Suppose that $y \in J_{x}^{+}(\omega)$. Then there exist sequences $\left\{t_{n}\right\}$ in $\mathbb{R}^{+}$and $\left\{x_{n}\right\}$ in $X$ with $x_{n} \rightarrow x$, $t_{n} \rightarrow+\infty$ and $\varphi\left(t_{n}, \theta_{-t_{n}} \omega\right) x_{n} \rightarrow y$. Set $\tau_{n}:=-t_{n}$ and $y_{n}:=\varphi\left(t_{n}, \theta_{-t_{n}} \omega\right) x_{n}$. Then $\left\{\tau_{n}\right\}$ is a sequence in $\mathbb{R}^{-}$with $\tau_{n} \rightarrow-\infty$ and $\left\{y_{n}\right\}$ is a sequence in $X$ and $y_{n} \rightarrow y$. 
Finlay we need to show that $\varphi\left(\tau_{n}, \theta_{-\tau_{n}} \omega\right) y_{n} \rightarrow x$.

$$
\begin{aligned}
& d\left(\varphi\left(\tau_{n}, \theta_{-\tau_{n}} \omega\right) y_{n}, x\right)= \\
& d\left(\varphi\left(\tau_{n}, \theta_{-\tau_{n}} \omega\right) \circ \varphi\left(t_{n}, \theta_{-t_{n}} \omega\right) x_{n}, x\right) \\
& \quad= \\
& d\left(\varphi\left(\tau_{n}, \theta_{-\tau_{n}} \omega\right) \circ \varphi\left(t_{n}, \theta_{-t_{n}} \omega\right) x_{n}, \varphi\left(\tau_{n}, \theta_{-\tau_{n}} \omega\right) y\right), \\
& \quad=d\left(\varphi\left(t_{n}, \theta_{-t_{n}} \omega\right) x_{n}, y\right) \rightarrow 0
\end{aligned}
$$

Then we have $\varphi\left(\tau_{n}, \theta_{-\tau_{n}} \omega\right) y_{n} \rightarrow x$.Thus $x \in J_{y}^{-}(\omega)$. Similarly we can prove the converse.

Theorem 5.11: $J_{x}^{+}\left(\theta_{t} \omega\right)=\varphi(t, \omega) J_{x}^{+}(\omega)$

Proof. To prove $J_{x}^{+}\left(\theta_{t} \omega\right)=\varphi(t, \omega) J_{x}^{+}(\omega)$.Let $z \in J_{x}^{+}\left(\theta_{t} \omega\right)$. Then there is a sequence $\left\{t_{n}\right\}$ in $\mathbb{R}^{+}$ with $t_{n} \rightarrow+\infty$ and a sequence $\left\{x_{n}\right\}$ in $X$ with $x_{n} \rightarrow x$ such that $\varphi\left(t_{n}, \theta_{-t_{n}} \theta_{t} \omega\right) x_{n} \rightarrow z$,

$$
\begin{gathered}
\varphi\left(t_{n}-t+t, \theta_{-t_{n}} \theta_{t} \omega\right) x_{n} \rightarrow z \\
\varphi(t, \omega) \varphi\left(t_{n}-t, \theta_{t-t_{n}} \omega\right) x_{n} \rightarrow z
\end{gathered}
$$

$\varphi\left(t_{n}-t, \theta_{t-t_{n}} \omega\right) x_{n} \rightarrow \varphi(t, \omega)^{-1} z \quad$,where $t_{n}-t$ $\rightarrow+\infty$.

Thus we have $\varphi(t, \omega)^{-1} z \in J_{x}^{+}(\omega)$. Then $\quad z \in$ $\varphi(t, \omega) J_{x}^{+}(\omega)$,

, then $J_{x}^{+}\left(\theta_{t} \omega\right) \subseteq \varphi(t, \omega) J_{x}^{+}(\omega)$. To prove the converse inclusion

Let $z \in \varphi(t, \omega) J_{x}^{+}(\omega)$. Then there is

$y \in J_{x}^{+}(\omega)$ with $z=\varphi(t, \omega) y$ and a sequence $\left\{t_{n}\right\}$ in $\mathbb{R}^{+}$with $t_{n} \rightarrow+\infty$ and a sequence $\left\{x_{n}\right\}$ in $X$ with $x_{n} \rightarrow x$ such that $\varphi\left(t_{n}, \theta_{-t_{n}} \omega\right) x_{n} \rightarrow y$. By the continuity of $\varphi(t, \omega)$, $\varphi(t, \omega) \circ \varphi\left(t_{n}, \theta_{-t_{n}} \omega\right) x_{n} \rightarrow \varphi(t, \omega) y$

$\varphi\left(t+t_{n}, \theta_{-t_{n}} \omega\right) x_{n} \rightarrow z$

$\varphi\left(t+t_{n}, \theta_{-t-t_{n}} \theta_{t} \omega\right) x_{n} \rightarrow z \quad$, where

$t_{n}+t \rightarrow+\infty, x_{n} \rightarrow x$.

Thus $z \in J_{x}^{+}\left(\theta_{t} \omega\right)$,we have

$\varphi(t, \omega) J_{x}^{+}(\omega) \subseteq J_{x}^{+}\left(\theta_{t} \omega\right)$.

Then $J_{x}^{+}\left(\theta_{t} \omega\right)=\varphi(t, \omega) J_{x}^{+}(\omega)$.

Theorem 5.12: If $\mathbb{X}$. is locally compact.Then $\Gamma_{M}^{+}(\omega) \neq \emptyset$ whenever $J_{M}^{+}(\omega)$ is non-empty and compact.

Proof. If possible let $\Gamma_{M}^{+}(\omega)=\emptyset$.Then we claim that $\gamma_{M}^{+}(\omega)$ is closed and disjoint with $J_{M}^{+}(\omega)$. That $\gamma_{M}^{+}(\omega)$ is closed follows from $\overline{\gamma_{M}^{+}(\omega)}=\gamma_{M}^{+}(\omega) \cup \Gamma_{M}^{+}(\omega)=\gamma_{M}^{+}(\omega)$ as $\Gamma_{M}^{+}(\omega)=\emptyset$, That $\Gamma_{M}^{+}(\omega) \cap J_{M}^{+}(\omega)=\emptyset$ follows from the fact that if $\Gamma_{M}^{+}(\omega) \cap J_{M}^{+}(\omega) \neq \emptyset$,then by invariance of $J_{M}^{+}(\omega), \Gamma_{M}^{+}(\omega) \subseteq J_{M}^{+}(\omega)$. Since $J_{M}^{+}(\omega)$ is compact ,we will have $\Gamma_{M}^{+}(\omega) \neq \varnothing$ and compact(remember that any sequence $\left\{y_{n}\right\}$ in a compact set $\mathrm{Q}$ has a convergent subsequence ). This again contradicts the assumption $\Gamma_{M}^{+}(\omega)=\varnothing$. Thus $\gamma_{M}^{+}(\omega)$ is closed and $\Gamma_{M}^{+}(\omega) \cap J_{M}^{+}(\omega)=$ $\emptyset$.Since $J_{M}^{+}(\omega)$ is non-empty and compact we have $d\left(\gamma_{M}^{+}(\omega), J_{M}^{+}(\omega)\right) \geq 0 \quad$. thus there is a $\quad r>0$ such that $S\left[J_{M}^{+}(\omega), r\right]$ is compact and disjoint with
$\gamma_{M}^{+}(\omega)$. Now choose any of $y \in J_{M}^{+}(\omega)$. There is a sequence $\left\{x_{n}\right\} \quad$ in $M\left(\theta_{-t_{n}} \omega\right)$ and a sequence $\left\{t_{n}\right\}$ in $R^{+}$such that $x_{n} \rightarrow x \in \cap M\left(\theta_{-t_{n}} \omega\right)$ and $t_{n} \rightarrow+\infty$ and, $\varphi\left(t_{n}, \theta_{-t_{n}} \omega\right) x_{n} \rightarrow y$. We may assume that $x \notin \gamma_{M}^{+}(\omega), \varphi\left(t_{n}, \theta_{-t_{n}} \omega\right) x_{n} \in S\left[J_{M}^{+}(\omega), r\right]$ for all $\mathrm{n}$.Then the trajectory segments $\varphi\left(s_{n}, \theta_{-s_{n}} \omega\right) x_{n}$ with $0 \leq s_{n} \leq t_{n}$, intersect $H\left(J_{M}^{+}(\omega), r\right)$ and therefor there is a sequence $\left\{\tau_{n}\right\}, 0 \leq \tau_{n} \leq t_{n}$, such that $\varphi\left(\tau_{n}, \theta_{-\tau_{n}} \omega\right) x_{n} \in H\left(J_{x}^{+}(\omega), r\right)$.Since $H\left(J_{x}^{+}(\omega), r\right)$ is compacte we may assume that $\varphi\left(\tau_{n}, \theta_{-\tau_{n}} \omega\right) x_{n} \rightarrow z \in$ $H\left(J_{x}^{+}(\omega), r\right)$.By taking subsequences we may assume that either $\tau_{n} \rightarrow t \in R^{+} \quad$ or $\tau_{n} \rightarrow+\infty$. If $\tau_{n} \rightarrow t$, then by the continuity axiom $\varphi\left(\tau_{n}, \theta_{-\tau_{n}} \omega\right) x_{n} \rightarrow$ $\varphi(t, \omega) x=z$,i.e, $z \in \gamma_{M}^{+}(\omega) \quad$ which contradicts $\gamma_{M}^{+}(\omega) \cap S\left[J_{M}^{+}(\omega), r\right]=\varnothing \quad$.If $\tau_{n} \rightarrow+\infty \quad$,then $z \in J_{M}^{+}(\omega)$,but this contradicts $z \in H\left(J_{x}^{+}(\omega), r\right)$ as $J_{M}^{+}(\omega) \cap H\left(J_{M}^{+}(\omega), r\right)=\varnothing$.

Theorem 5.13. Let $\mathbb{X}$. be locally compact. Then $J_{M}^{+}(\omega)$ is non -empty

And compact if and only if $D_{M}^{+}(w)$ is compact.

Proof. Let $J_{M}^{+}(\omega)$ be non -empty and compact.Then $\Gamma_{M}^{+}(\omega)$ is non empty and compact .But then $\overline{\gamma_{M}^{+}(\omega)}$ is $\operatorname{compact} \overline{\gamma_{M}^{+}(\omega)}$ is closed with $\mathbb{X}$ be locally compact).Hence $D_{M}^{+}(w)=\gamma_{M}^{+}(\omega) \cup J_{M}^{+}(\omega)$

$=\overline{\gamma_{M}^{+}(\omega)} \cup J_{M}^{+}(\omega)$ is compact .Now $D_{M}^{+}(w)$ is compact. Since $J_{M}^{+}(\omega) \subseteq D_{M}^{+}(w)$.Then $J_{M}^{+}(\omega)$ is compact.

Theorem 5.14: If $\mathbb{X}$ is locally compact. Then $J_{M}^{+}(\omega)$ is connected.

Proof: Let $J_{M}^{+}(\omega)$ be compact . If $J_{M}^{+}(\omega)=\varnothing$ there is nothing to prove. So let $J_{M}^{+}(\omega) \neq \varnothing$.If $J_{M}^{+}(\omega)$ is disconnected, then there are non-empty compact sets $P, Q$ such that $J_{M}^{+}(\omega)=P \cup Q$ and $=P \cap Q=\emptyset$.Since $\Gamma_{M}^{+}(\omega)$ is non- empty and compact ,hence connected, we have $\Gamma_{M}^{+}(\omega) \subset P$ or $\Gamma_{M}^{+}(\omega) \subset Q$. Let $\Gamma_{M}^{+}(\omega) \subset P \quad . \operatorname{Since} \gamma_{M}^{+}(\omega) \cup P=\overline{\gamma_{M}^{+}(\omega)} \cup P$ as $\Gamma_{M}^{+}(\omega) \subset P$ and $\overline{\gamma_{M}^{+}(\omega)} \quad$ is compact. Then $\gamma_{M}^{+}(\omega) \cup P$ is compact. Now let $Q \cap\left(\gamma_{M}^{+}(\omega) \cup P\right) \neq \varnothing$ $\left(Q \cap \gamma_{M}^{+}\right) \cup(Q \cap P) \neq \emptyset \quad$,then $Q \cap \gamma_{M}^{+} \neq \varnothing$.But $Q$ must be invariant. Thus will show that $\Gamma_{M}^{+}(\omega) \subset$ $Q$,aconradiction. Then $\gamma_{M}^{+}(\omega) \cup P$ is compact and disjoint from $Q, D_{M}^{+}(w)=\gamma_{M}^{+} \cup J_{M}^{+}(\omega)=\left(\gamma_{M}^{+}(\omega) \cup\right.$ $P) \cup Q$.since $\gamma_{M}^{+}(\omega) \cup P$ and $Q$ are disjoint compact sets we have $D_{M}^{+}(w)$ is disconnected. Thus is a contradiction . Then $J_{M}^{+}(\omega)$ is connected.

\section{Conclusion}

This paper has been studied the concept of Prolongation Limit Random Sets in Random Dynamical Systems. we prove that the First Prolongation of a closed random set containing this set, the First Prolongation is closed and invariant, also it is connected whenever it is compact provided that the phase space of the RDS is locally compact. Then we study the Prolongational Limit Set for RDS and proved some essential properties of this set. 
Where we prove that the Prolongational Limit Set for RDS is closed and invariant. Also the relation among the the First Prolongation, the Prolongational Limit Set and the positive trajectory of a random set is given and proved. Also if the phase space of RDS is locally compact then the following statements are true : if the Prolongational Limit Set for RDS is nonempty and compact, then the omega-limit set is non-empty; the Prolongational Limit Set for RDS is nonempty and compact if and only if the the First Prolongation is compact. Finally the Prolongational Limit Set for RDS is connected.

\section{References:}

[1]S.M. Ulam and J. Von Neumann,"Random Ergdic Theorem ,Bull. Amer.Mth. Soc.51(1945)).

[2] L. Arnold and I.D. Chueshov,"Order -Preserving Random Dynamical Systems: Equilibria, attractor applications", Dynamics and Stability of Systems, 13(1998), 265-280 .

[3] D. T. Son," Lyapunov Exponents for Random Dynamical Systems", Phd thesis der Fakult"at Mathematik und Naturwissenschaften der Technischen Universit“at Dresden (2009).

[4] X. Yingchao, " The Random Attractors of Stochastic Duffing-Van Der Pol Equations with Jumps", Chinese Journal of Applied Probability and Statistics 26(1) Feb (2010).

[5] I.J.Kadhim and A.H. Khalil," On Expansive Random Operators over a Uniform Random Dynamical Systems", European Journal of Scientific Research, 142 ( 4) October pp.334-342 (2016)
[6] Chuanxi Zhu and Chunfang Chen," Calculations of random fixed point index". J. Math. Anal. Appl. 339 (2008) 839-844.

[7] H.E. Kunze D. La Torreb and E.R. Vrscay," Random fixed point equations and inverse problems using "collage method" for contraction mappings",J. Math. Anal. Appl. 334 (2007) 1116-1129.

[8] Ismat Beg and Mujahid Abbas," Random fixed point theorems for a random operator on an unbounded subset of a Banach space",Applied Mathematics Letters 21 (2008) 1001-1004.

[9] L. Arnold, "Random dynamical systems". Springer, Berlin (Corrected 2nd printing), (2003).

[10] I. Chueshov " Monotone Random Systems Theory and Applications" Springer- Verlag Berlin Heidelberg Germany (2002) 31-38.

[11] H. Crauel, Random point attractors versus random set attractors, J. London Math. Soc., II. Ser., 63 (2001) 413 427.

[12] T. Ura, Sur les courbes définies par les équations différentielles dans I'espace à $m$ dimensions, Ann. Sci. École Norm. Sup. 70 (1953) 287-360.

[13] N.P. Bhatia, G.P. Szegö, "Stability Theory of Dynamical Systems", Springer-Verlag, Berlin.( 1970). 


\section{بعض الخصائص مجموعات الغايه المستطيله العشوائيه في النظام الايناميكي العشوائي المبني \\ سنلس طالب محسن احسان جبار كاظم \\ قسم الرياضيات ، كلية علوم الحاسوب والرياضيات ، جامعة القادسية}

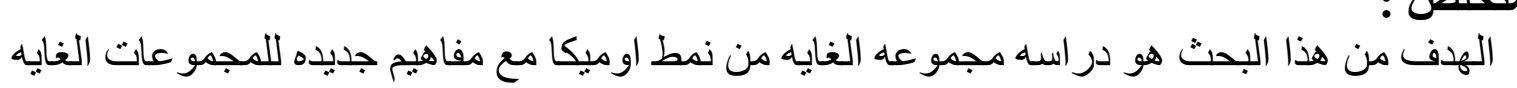

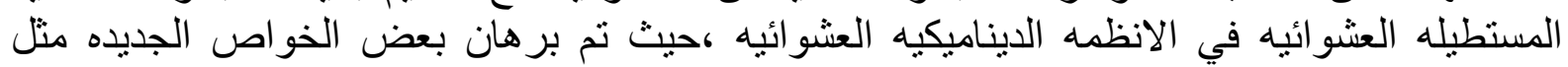

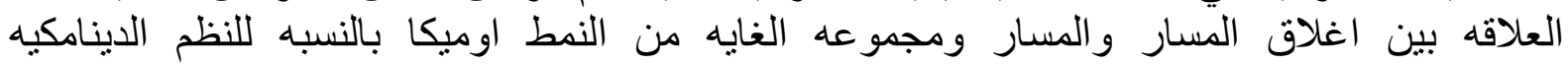

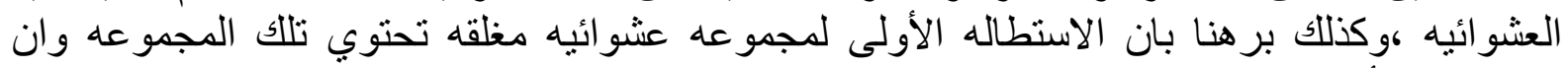

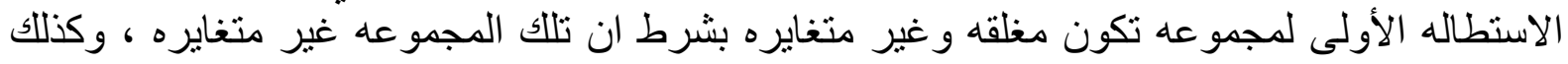

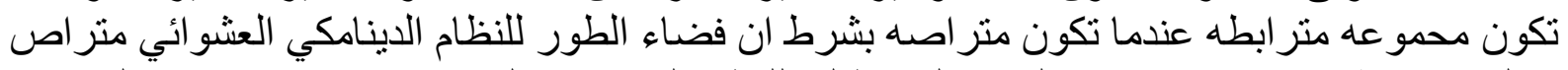

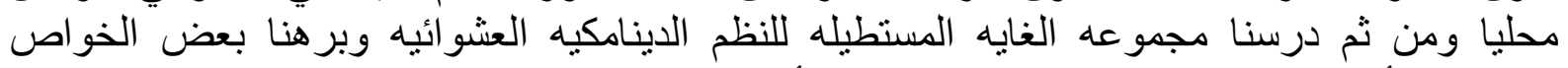
الاساسيه،و أخيرا بر هنا العلاقه بين الاستطاله الأولى ومجموعه الغايه المستطيله و المسار الموجبه لئه لمجمو عه عشوائيه. 